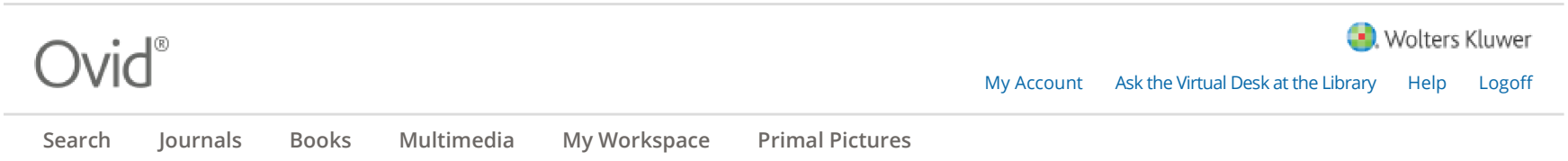

\title{
Annals of Surgery
}

Issue: Volume 223(3), March 1996, pp 242-248

Copyright: @ Lippincott-Raven Publishers.

Publication Type: [Review Article]

ISSN: 0003-4932

Accession: 00000658-199603000-00003

\section{Foreign Material in Postoperative Adhesions}

Luijendijk, R. W. M.D.*; de Lange, D. C.D. M.D.; Wauters, C. C.A.P. M.D. ${ }^{\dagger}$; Hop, W. C.J. ${ }^{\ddagger} ;$ Duron, J. J. ${ }^{[/ /]}$; Pailler, J. L. ";

Camprodon, B. R..\#; Holmdahl, L."; van Geldorp, H. J. ${ }^{\text {s. }}$ Jeekel, J.*

\section{Author Information}

From the Departments of General Surgery, ${ }^{\star}$ Pathology, ${ }^{\dagger}$ Epidemiology \& Biostatistics, ${ }^{\ddagger}$ and Gynecology, ${ }^{\S}$ University Hospital Rotterdam Dijkzigt, Rotterdam, The Netherlands; the Department of General Surgery, Groupe Hospitalier Pitié-Salpétrière, ${ }^{[/ /]}$ Paris, France; the Department of General Surgery, Hôpital d'Instruction des Armées du Val-de-Grâce, " Paris, France; the Department of General Surgery, Hospital de la Esperanza, "Barcelona, Spain; and the Department of General Surgery,

University of Göteborg Östra Hospital," Göteborg, Sweden

Address reprint requests to J. Jeekel, Professor of Surgery, University Hospital Rotterdam-Dijkzigt, Dr. Molewaterplein 40, 3015 GD Rotterdam, The Netherlands.

Accepted for publication March 10, 1995.

\section{Abstract}

Objective: The authors determined the prevalence of foreign body granulomas in intra-abdominal adhesions in patients with a history of abdominal surgery.

Patients and Methods: In a cross-sectional, multicenter, multinational study, adult patients with a history of one or more previous abdominal operations and scheduled for laparotomy between 1991 and 1993 were examined during surgery. Patients in whom adhesions were present were selected for study. Quantity, distribution, and quality of adhesions were scored, and adhesion samples were taken for histologic examination.

Results: In 448 studied patients, the adhesions were most frequently attached to the omentum (68\%) and the small bowel $(67 \%)$. The amount of adhesions was significantly smaller in patients with a history of only one minor operation or one major operation, compared with those with multiple laparotomies $(\mathrm{p}<0.001)$. Significantly more adhesions were found in patients with a history of adhesions at previous laparotomy $(\mathrm{p}<0.001)$, with presence of abdominal abscess, hematoma, and intestinal leakage as complications after former surgery $(p=0.01, p=0.002$, and $p<0.001$, respectively), and with a history of an unoperated inflammatory process $(p=0.04)$.

Granulomas were found in $26 \%$ of all patients. Suture granulomas were found in $25 \%$ of the patients. Starch granulomas were present in $5 \%$ of the operated patients whose surgeons wore starch-containing gloves. When suture granulomas were present, the median interval between the present and the most recent previous laparotomy was 13 months. When suture granulomas were absent, this interval was significantly longer - i.e., 30 months $(p=0.002)$. The percentage of patients with suture granulomas decreased gradually from $37 \%$ if the previous laparotomy had occurred up to 6 months before the present operation, to $18 \%$ if the previous laparotomy had occurred more than 2 years ago $(p<0.001)$.

Conclusions: The number of adhesions found at laparotomy was significantly larger in patients with a history of multiple laparotomies, unoperated intraabdominal inflammatory disease, and previous postoperative intra-abdominal complications, and when adhesions were already present at previous laparotomy. In recent adhesions, suture granulomas occurred in a large percentage. This suggests that the intra-abdominal presence of foreign material is an important cause of adhesion formation. Therefore intra-abdominal contamination with foreign material should be minimized.

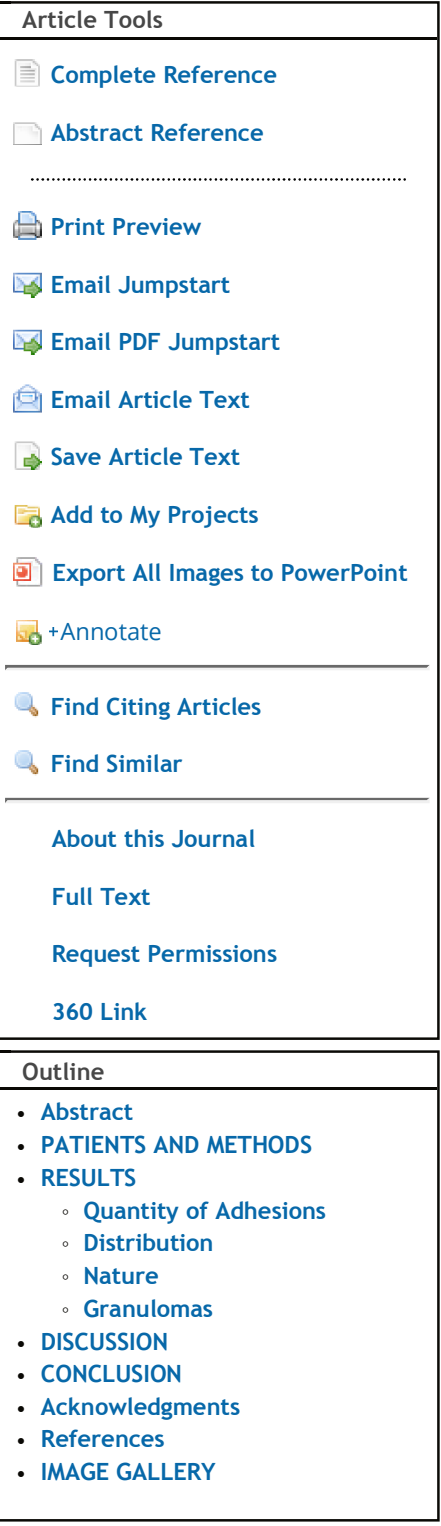


has been estimated that one third of intestinal obstructions $1-5$ and $15 \%$ to $20 \%$ of female infertility is caused by adhesions.6,7 The amount of effort general surgeons put into work because of adhesions is large. Approximately $1 \%$ of all surgical admissions and $3 \%$ of laparotomies are the result of intestinal obstruction from adhesions. 2 The treatment of patients with symptoms caused by adhesions also will generate extra costs.

Adhesions are congenital or develop after an abdominal operation or infection. 2,3 Intraoperative tissue damage, infections, tissue ischemia, and intraabdominal presence of foreign material, blood, or bile,1,8-15 all have been shown to be potent causes of peritoneal adhesions. Foreign materials, such as glove powder, 11 fluff from surgical packs (gauze lint), 12 sutures, 13,14 and material extruded from the digestive tract, cause a peritoneal inflammatory reaction. 15 This reaction potentiates adhesion formation, especially with concomitant peritoneal damage, as has been demonstrated in various animal models. 1,11,13,15-18 Such adhesions often contain multiple foreign body granulomas.10,16,17,19-23 This strongly suggests a relation between foreign material, foreign body granulomas, and adhesion formation. Foreign bodies contaminating the peritoneal tissues also might be a cause of adhesion formation in humans. Reported studies are small or date from the time when the contemporary advanced hygienic surgical techniques were not in use.24-26 In the current series, the presence of foreign body granulomas and adhesions after laparotomy was studied in humans.

Back to Top

\section{PATIENTS AND METHODS}

Adult patients with a history of previous abdominal surgery and scheduled for laparotomy between 1991 and 1993 at the University Hospital Rotterdam (Rotterdam, The Netherlands = NL), Groupe Hospitalier Pitié-Salpétrière (Paris, France = F1), Hôpital d'Instruction des Armées du Val-de-Grâce (Paris, France = F2), Hospital de la Esperanza (Barcelona, Spain = SP), and the University of Göteborg Östra Hospital (Göteborg, Sweden = SW), were examined during surgery. Patients were selected for study if adhesions appeared to be present. During the operation, the quantity, distribution, and quality of adhesions were scored, and adhesion samples were taken for histologic examination. Quantity was grouped as 1 to 3, 4 to 10 , and more than 10 adhesions. 23 To score distribution, adhesion adherence to the following structures was determined: laparotomy scar, omentum, abdominal wall, liver, spleen, stomach, small intestine, colon, retroperitoneum, and in female reproductive organs. Whenever structures were absent or not explored, this was noted. In addition, adhesions were scored using the macroscopic classification according to Zühlke (Table 1).27 One to three samples of adhesions were taken. These samples were fixed in $4 \%$ formaldehyde, put in paraffin, sectioned (six micron), and stained with hematoxylin and eosin (H\&E) and periodic acid Schiff's reagens. Then, the adhesions were scored microscopically, using the histologic classification according to Zühlke (Table 2),27 and the slides were screened for granulomas and foreign material in or near histiocytes or giant cells, using both normal and polarized light. Using polarized light, glove starch powder can be recognized by the typical birefringent Maltese crosses.

Patient-related factors of gender, age, history of previous abdominal surgery, radiotherapy, and prior inflammatory disease and operation-related factors including the presence of adhesions at prior surgery, postoperative complications (abscess, hemorrhage, anastomotic bowel leakage), use of prosthetic material, and use of powdered or unpowdered gloves were analyzed. An appendectomy, hysterectomy, incisional hernia repair, or diagnostic laparotomy was scored as minor operation. All other abdominal operations were scored as major operations.

Statistical methods used included the Kruskas Wallis test or the Mann-Whitney test for the comparison of ordered classifications and time intervals. Percentages were compared using the chi square test. Logistic regression 28 was used to evaluate various factors simultaneously with regard to the percentage of patients who had more than ten adhesions. This technique also was used to assess the relation between the percentage of patients with granulomas and the (logarithmically transformed) time interval from the previous operation. $P$ values given are two-sided; 0.05 was considered the limit of significance.

Back to Top

RESULTS

Back to Top Quantity of Adhesions

A total of 448 patients with adhesions was included (222 NL, 139 F1, 14 F2, 44 SP, 29 SW). There were 202 men and 246 women; $27 \%$ had 1 to 3 intra-abdominal 
adhesions, $30 \%$ had 4 to 10 intra-abdominal adhesions, and $43 \%$ had more than 10 intra-abdominal adhesions. A history of one minor, one major, or multiple operations was present in $24 \%, 33 \%$, and $43 \%$ of the patients, respectively.

The number of adhesions was significantly lower in patients with a history of only one minor or one major operation, compared with those with multiple laparotomies $(p<0.001)$. No significant difference was found between one minor and one major operation $(p=0.15$, Table 3$)$.

The number of adhesions was significantly larger in patients with adhesions at previous laparotomy (Table 4) compared with those without $(p<0.001)$. Also, significantly more adhesions were found in patients with a history of a surgical complication, such as abdominal abscess $(p=0.01)$, hematoma $(p=0.002)$ and intestinal leakage $(p<0.001)$, compared with patients who had an uncomplicated course. Significantly more adhesions also were found in patients with a history of an unoperated inflammatory process compared with patients without $(p=0.04)$. No significant difference in amount of adhesions was found between patients who had prosthetic material intra-abdominally or in those who had received abdominal radiotherapy.

Using multivariate analysis, it was found that, besides the type of previous operation and the presence of adhesions at previous operation, intestinal leakage was the most important factor regarding the presence of more than ten adhesions. None of the other factors that were significantly related to the amount of adhesions when considered alone (abdominal abscess, hematoma and previously unoperated inflammatory process) appeared of importance when taking into account the presence of adhesions and intestinal leakage at previous operations and type of previous operation.

Back to Top

Distribution

In calculating the percentages of patients with adhesions per organ, patients were excluded if the organ was not visualized during surgery. The distribution was as follows: omentum, 293 of 430 patients (68\%); small bowel, 288 of 428 patients (67\%); abdominal wall, 193 of 432 patients (45\%); female reproductive organs, 49 of 212 patients (23\%); colon, 205 of 419 patients (41\%); liver, 118 of 352 patients (34\%); stomach, 67 of $342(20 \%)$; retroperitoneum, 56 of 388 patients $(14 \%)$; and spleen, 28 of 309 patients (9\%). The laparotomy scar was attached to one or more of the following organs in 314 out of 440 cases (71\%): omentum, small bowel, colon, or abdominal wall. The site of previous surgery was attached to one or more of these organs in 229 of 434 cases (53\%). Remote adhesions (to structures not previously operated on) were adherent to one or more of these organs in 156 of 436 cases (35\%).

Back to Top

Nature

Of 24 patients with adhesions, either no biopsy material or unsuitable biopsy material was taken, leaving 424 patients for microscopic examination. The percentages of adhesions scored in the macroscopic $(n=429)$ and histologic $(n=$ 415) Zühlke classification I, II, III, and IV were, respectively, $11 \%, 30 \%, 49 \%$, and $10 \%$ and $7 \%, 49 \%, 37 \%$, and $7 \%$. No relationship was found between 1) both classifications and 2 ) the time interval to the most recent operation and presence of granulomas.

Back to Top

Granulomas

Granulomas were found in 112 of 424 patients (26\%). Suture granulomas (Fig. 1) were found in 105 of 424 patients (25\%). Starch granulomas (Fig. 1) were found in 14 of 309 patients $(5 \%)$ with a history of being operated on with starch-containing gloves.

In those patients with suture granulomas $(\mathrm{n}=105)$, the median interval between the present and previous operation was 13 months. This interval was significantly longer $(p=0.002)$ for patients with no suture granulomas (median 30 months). Table 5 shows the percentage of patients with suture granulomas according to the interval from the previous operation. The percentage decreased gradually from $37 \%$ if the previous operation had occurred up to 6 months before the present operation, to $18 \%$ if the previous operation had occurred more than 2 years ago. Figure 2 shows the percentages according to the interval on a continuous basis.

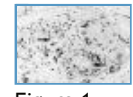

Figure 1

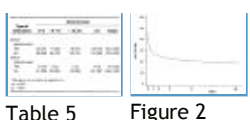

Table 5 Figure 2 
In patients with starch granulomas $(n=14)$, the median interval between the present and previous operation was 14 months. This interval showed a trend to be longer $(p=0.09)$ for patients with no suture granulomas with a median of 42 months. The percentage of patients with starch granulomas according to the interval from previous operation also is shown in Table 5.

Back to Top

\section{DISCUSSION}

More than 25 years ago, Myllärniemi reported that $61 \%$ of 309 patients with postoperative adhesions showed reactions to foreign material. Substances identified included talc (31\%), gauze lint (16\%), a combination of talc and gauze lint $(11 \%)$, starch $(1 \%)$, and sutures $(<1 \%)$. In contrast, in the current series $25 \%$ of 424 patients had suture granulomas, and $5 \%$ of 309 patients being operated on with starch containing gloves had starch granulomas. This difference might be explained partially by the stringent criteria used for identification of foreign body granulomas, which reduces the chance of contamination being erroneously scored as such. Further, talc glove powder no longer is in use, and has been replaced by starch. Another explanation might be a different distribution of patients with respect to the interval between the present and the most recent previous operation. Foreign body granulomas are found more often in patients recently operated on. In the current study, this was demonstrated for suture granulomas, but not for starch granulomas. An explanation for this difference might be the ability of the body to resorb starch and suture materials. The largest extent of this resorption of suture materials seems to take place during the first year.

The number of granulomas found at laparotomy might be even larger than accounted for in the current study. First, in patients with a history of multiple laparotomies, the adhesion removed for microscopic examination might have formed before the most recent operation. This reduces the chance of finding granulomas because of resorption. Second, granulomas might be present in the adhesion but not in the sample or histologic slide. Third, resorption of foreign material might have been completed, leaving an empty granuloma or, when the granuloma itself also has disappeared with time, an adhesion without granulomas. 15 This resorption of foreign material, however, comes too late to prevent adhesion formation. The organization of fibrinous adhesions starts at 3 days. 29

The resorption rate strongly influences the chance of finding foreign material. The resorption rate of starch powder is not known, but will depend on the glove powder used (kind, composition, amount, clumping) and the host (intra-abdominal conditions, individual inflammatory reaction/sensitivity). 23 Experiments on animals suggest that powder can be resorbed within 24 hours, leaving granulomas and firm adhesions long thereafter.16 At the latest, starch granulomas still were present at 15 months in experimental studies 11 and at 23 months in clinical studies. 24 In the current study, starch granulomas were found mostly up to 48 months. However, in four patients, starch granulomas were found much later-up to 32 years after the last operation. We do not have an explanation for this finding.

The question remains: why were suture granulomas found so often in this study (105/424; 25\%), whereas in other studies, almost no suture granulomas were found? Specimens were taken at random, and the presence of a granuloma or suture seldomly was suspected macroscopically. Remarkably, in 39 of 211 patients (18\%) who had the last operation more than 2 years previously versus 66 of 213 (31\%) who had had their last operation less than 2 years previously, suture granulomas were present. Therefore, the large amount of suture granulomas cannot be explained exclusively by use of nonabsorbable suture material. Unfortunately, we were not able to differentiate between absorbable or nonabsorbable suture in our material.

Apart from the aforementioned, the presence of suture material and the ischemia caused by tightening the suture can potentiate adhesion formation.23,30 This may explain why the laparotomy scar so often is attached to its surroundings by adhesions.

As a consequence to these results, the use of suture material should be minimized to avoid the intra-abdominal presence, as well as the ischemia it causes. Closure of the peritoneum, for instance, is unnecessary 1,29 and in the aforementioned context, unwanted. A meticulous technique can limit foreign body contamination and subsequent granuloma formation and peritoneal damage. This may reduce adhesion formation. 5

Powder-free gloves prevent starch granulomas, and thus are recommended. Powdered gloves can be made powder free by a 1-minute washing with $10 \mathrm{~mL}$ of 


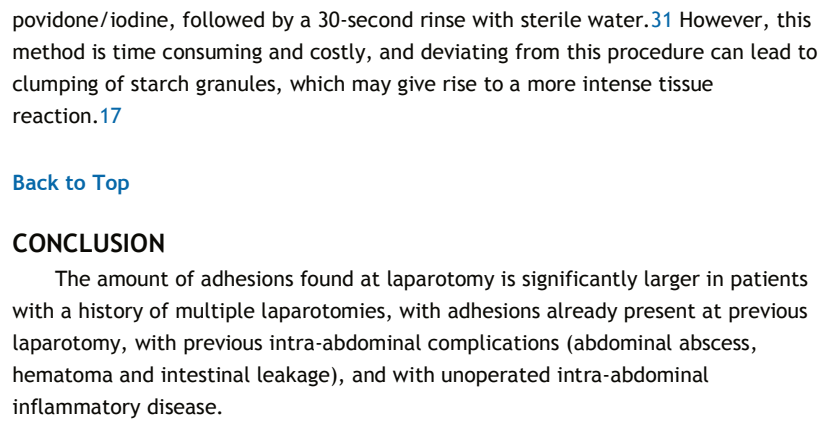

\section{CONCLUSION}

The amount of adhesions found at laparotomy is significantly larger in patients with a history of multiple laparotomies, with adhesions already present at previous laparotomy, with previous intra-abdominal complications (abdominal abscess, hematoma and intestinal leakage), and with unoperated intra-abdominal inflammatory disease.

Microscopic examination of adhesions showed granulomas in a large percentage (26\%), with suture granulomas in $25 \%$ and starch granulomas in $5 \%$. Suture granulomas were found significantly more often in patients recently undergoing surgery. Therefore, modern surgical techniques are not as meticulous as we might think. If the causative relation between foreign material, foreign body granulomas, and adhesion formation shown in animals also exists in humans, the operative contamination with foreign material is an important cause of adhesion formation. In view of the serious consequences, such as intestinal obstruction and infertility, prevention of contamination during surgery with foreign material is of considerable importance.

Back to Top

\section{Acknowledgments}

The authors thank Professor H. Ellis of the University of London for the careful and critical reading of the manuscript, and the valuable advice.

Back to Top

\section{References}

1. Ellis H. The Causes and prevention of intestinal adhesions. Br J Surg 1982 69:241-243. 360 Link| Bibliographic Links|[Context Link]

2. Menzies $\mathrm{D}$, Ellis $\mathrm{H}$. Intestinal obstruction from adhesions-how big is the problem? Ann R Coll Surg Engl 1990; 72:60-63. [Context Link]

3. Menzies D. Peritoneal adhesions: incidence, cause and prevention. Surg Annu 1992; 24:27-45. 360 Link| Bibliographic Links|[Context Link]

4. McEntee G, Pender D, Mulvin D, et al. Current spectrum of intestinal obstruction. Br J Surg 1987; 74:976-80. 360 Link| Bibliographic Links| [Context Link]

5. Keeman JN. Recurrent obstructive ileus due to adhesions. Neth J Med 1987; 131:1509-1512. 360 Link| [Context Link]

6. Fayez JA. An assessment of the role of operative laparoscopy in tuboplasty. Fertil Steril 1983; 39:476-479. 360 Link| Bibliographic Links| [Context Link]

7. Hershlag A, Diamond MP, DeCherney AH. Adhesiolysis. Clin Obstet Gynecol 1991; 34:395-402. Ovid Full Text 360 Link| Bibliographic Links|[Context Link]

8. Ellis $\mathrm{H}$. The cause and prevention of postoperative intraperitoneal adhesions. Surg Gynecol Obstet 1971; 133:497-511. [Context Link]

9. Almdahl SM, Burhol PG. Peritoneal adhesions: causes and prevention. Dig Dis 1990; 8:37-44. [Context Link]

10. Myllärniemi $H$, Frilander $M$, Turunen $M$, Saxén $L$. The effect of glove powders and their constituents on adhesion and granuloma formation in the abdominal cavity of the rabbit. Acta Chir Scan 1966; 131:312-318. 360 Link| Bibliographic Links | [Context Link]

11. Ellis $\mathrm{H}$. The hazards of surgical glove dusting powders. Surg Gynecol Obstet 1990; 171:521-527. 360 Link| Bibliographic Links|[Context Link]

12. Down RHL, Whitehead R, Watts JMcK. Do surgical packs cause peritoneal adhesions? Aust NZ J Surg 1979; 49:379-382. 360 Link| Bibliographic Links [Context Link] 


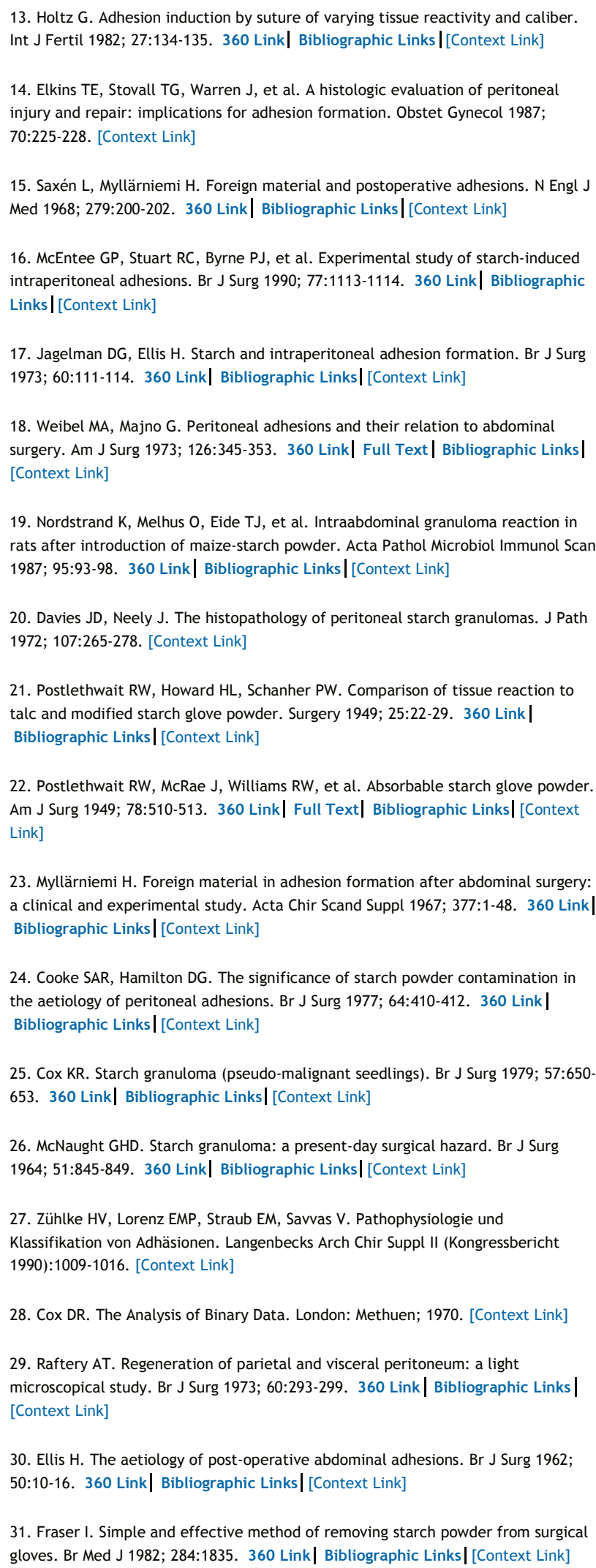

25. Cox KR. Starch granuloma (pseudo-malignant seedlings). Br J Surg 1979; 57:650653. 360 Link| Bibliographic Links| [Context Link]

26. McNaught GHD. Starch granuloma: a present-day surgical hazard. Br J Surg 1964; 51:845-849. 360 Link| Bibliographic Links| [Context Link]

27. Zühlke HV, Lorenz EMP, Straub EM, Savvas V. Pathophysiologie und Klassifikation von Adhäsionen. Langenbecks Arch Chir Suppl II (Kongressbericht 1990):1009-1016. [Context Link]

28. Cox DR. The Analysis of Binary Data. London: Methuen; 1970. [Context Link]

29. Raftery AT. Regeneration of parietal and visceral peritoneum: a light microscopical study. Br J Surg 1973; 60:293-299. 360 Link| Bibliographic Links | [Context Link]

30. Ellis $\mathrm{H}$. The aetiology of post-operative abdominal adhesions. Br J Surg 1962; 50:10-16. 360 Link| Bibliographic Links| [Context Link]

31. Fraser I. Simple and effective method of removing starch powder from surgical gloves. Br Med J 1982; 284:1835. 360 Link| Bibliographic Links| [Context Link]

\section{IMAGE GALLERY}

Select All 


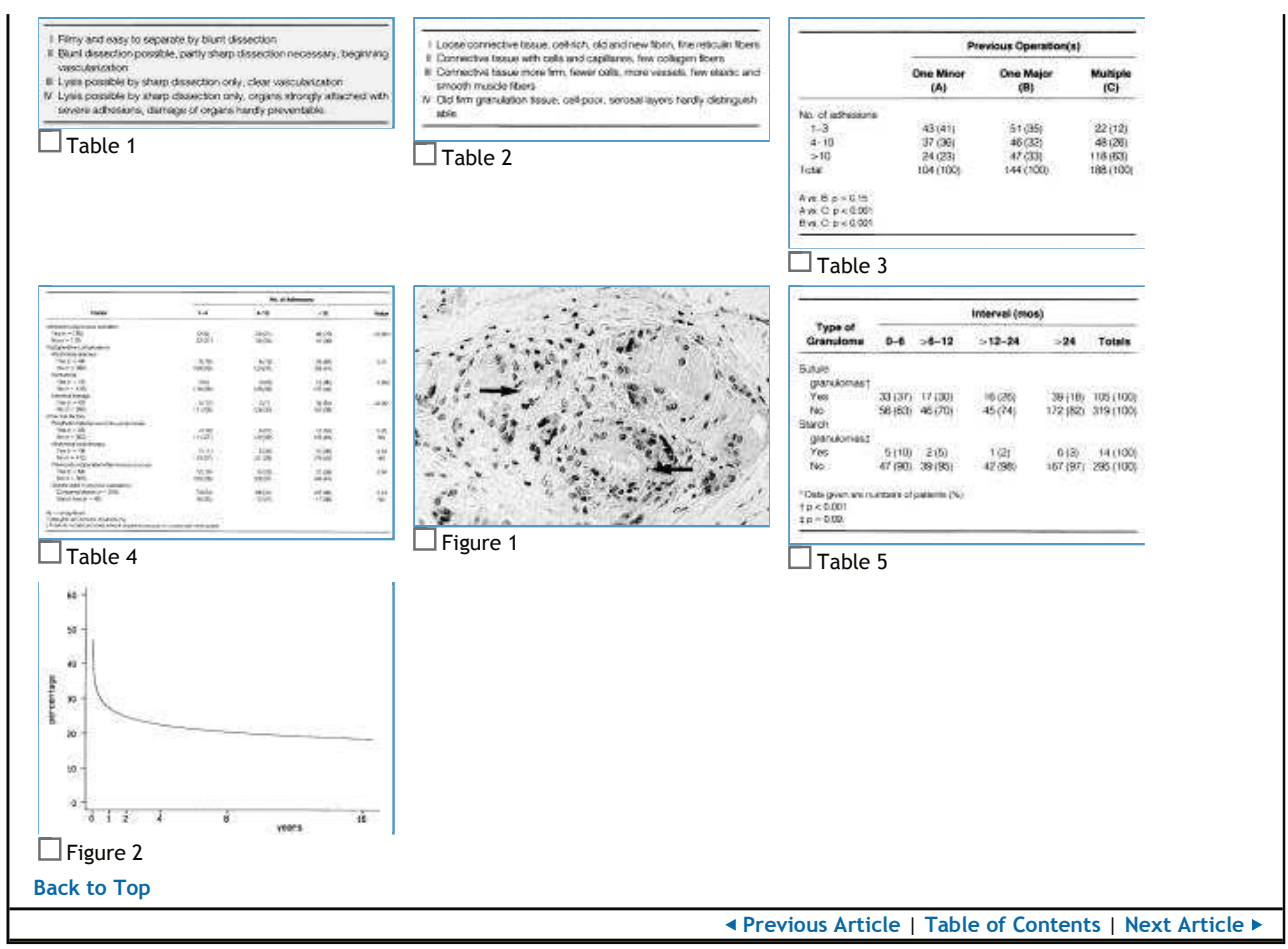

(c) 2015 Ovid Technologies, Inc. All rights reserved. OvidSP_UI03.15.01.102, SourcelD 66680 